\title{
Nonparametric Smooth Estimation of Probability Density Function and Other Related Functionals: Some New Developments
}

\author{
Yogendra P. Chaubey \\ Department of Mathematics and Statistics Concordia University, Montreal, (QC) Canada H3G 1M8 \\ yogen.chaubey@concordia.ca
}

In this talk I will highlight some recent developments in the area of nonparametric functional estimation with emphasis on nonparametric density estimation. A lemma attributed to Hille, and its generalization [see Lemma 1, Feller (1965) An Introduction to Probability Theory and Applications, §VII.1)] has been used to propose estimators in the context of i.i.d. observations in Chaubey and Sen (1996, Statistics and Decisions) and Chaubey et al. (2012, Jour. Ind. Stat. Assoc.). The generality of the technique will be illustrated to non-standard situations such as biased data and circular data. A review of recent results and contrasting various techniques will also be presented. 